\title{
Analysis of a novel comprehensive health system
}

\author{
Mehdi Rezaei Aderyani \\ Department of Pardis, Ferdowsi University of Mashhad, Mashhad, Iran
}

\begin{abstract}
So far, different definitions of health systems have been provided by international health organizations in which it has been tried to show the capabilities of a health system. Of course a system that have all these capabilities has never been offered because these capabilities are associated with different health areas including telemedicine, and disease diagnosis, and there is no system that is capable of gathering all these features. In this study we attempt to define a system that meets the information needs a comprehensive health system at once; a system which covers most of the health areas, including medical, food and medicine. In this article we are going to discuss the most important capabilities that a comprehensive health system has. This application will bring economic efficiency for its users, because with its help, people can easily distinguish their body's needs based on specific circumstances and act accordingly. This will prevent many food caused diseases. Also, people using this application will not need to spend heavy expenses to prepare healthy food and they can prepare healthy foods with the help of healthy foodstuffs; as we know in many developed countries, to prevent food caused diseases, people have turned to diets such as vegetarian diet so they don't suffer such diseases due to inactivity. Users of this application can cook or prepare foods proposed by system with the items and healthy food and with the help of search program that is available in the system, and receive the most information about that food.
\end{abstract}

KEY WORDS: COMPREHENSIVE HEALTH SYSTEM, HEALTH CARE SYSTEM, DISEASE DIAGNOSIS SYSTEM

\section{INTRODUCTION}

Food is almost one of the basic human needs and according to its fundamental role in providing health, extensive research has been done about it. In this article we are going to discuss the most important capabilities that a comprehensive health system has. This application will bring economic efficiency for its users,

\section{ARTICLE INFORMATION:}

*Corresponding Author:8512713@gmail.com Received $27^{\text {th }}$ Dec, 2017

Accepted after revision $21^{\text {st }}$ March, 2018

BBRC Print ISSN: 0974-6455

Online ISSN: 2321-4007 CODEN: USA BBRCBA

$\therefore$ Thomson Reuters ISI ESC / Clarivate Analytics USA and Crossref Indexed Journal

NAAS Journal Score 2017: 4.38 SJIF 2017: 4.196

- A Society of Science and Nature Publication, Bhopal India 2018. All rights reserved.

Online Contents Available at: http//www.bbrc.in/

DOI: $10.21786 / \mathrm{bbrc} / 11.1 / 21$ because with its help, people can easily distinguish their body's needs based on specific circumstances and act accordingly. This will prevent many food caused diseases. Also, people using this application will not need to spend heavy expenses to prepare healthy food and they can prepare healthy foods with the help of healthy foodstuffs; as we know in many developed countries, to prevent food caused diseases, people have turned to 
diets such as vegetarian diet so they don't suffer such diseases due to inactivity. Users of this application can cook or prepare foods proposed by system with the items and healthy food and with the help of search program that is available in the system, and receive the most information about that food,( Health Systems, Goals, 22 January 2017).

The information system of this application is of database type and in the form of entity, (September 2005, http://whatis.techtarget.com/definition/entity), and relational or tabular. This product is a site to which the applicants join by paying a sum of money and use the site services and since a system with this characteristics and comprehensiveness does not exist and also it resolves the basic needs of the people, it most likely will be successful in the market and will have a good sale in the case of good and sufficient advertising, (Health systems, Goals, 22 January 2015).

Today, a high diversity of health systems are produced and each of them are somehow trying to solve medical and public health problems or provided facilities to fix the problems. According to the World Health Organization, health systems goals must be appropriately and equitably available to public, they also should have good, effective, and acceptable quality and evenly distributed for the general public, (health systems, World Health Organization definition, 22 January 2015,http:// en.wikipedia.org/wiki/Health_system). These cases also known as 5C in the United States which include: Cost, Coverage, Consistency, Complexity, and Chronic Illness, (Health systems ,Goals, 22 January 2015).

\section{SUMMARY OF MANAGEMENT TOPICS}

The project is an application that is produced in the form of a website. The totality of project is as follows:

The product is an application relating to nutrition, health and wellness; a product, which provides comprehensive information about cooking, medicine and health and solves many problems that have been discussed below. Recently, people should not use food only for feeding. We argue that the proper use of this system will increase the people life to one hundred years. In fact, it is the system that decides what organic matter and how much energy and vitamins your body needs and therefore what foods you should consume. For this purpose, system receives complete information about your body and diseases that you likely suffer and the amount of your daily physical activity and sport and many other daily activities you were indifferent towards them so far and it also examines where you live in terms of weather and according to these information, teaches you the healthy lifestyle and how do stay healthy and warns you the necessary medical advices.
Some people do not want the system decides about their food and wish to choose their own food. This system has predicted this, too. The system has a complete list of a variety of dishes at once and allows you, for example, to prepare new and local dishes for your family according to the food in your cabinets and fridge. You can ask the system to analyze these foods for you and thus you will know that what food you have on table and eat and how much energy it gives to your body, or how much vitamins, fat, carbohydrates and other organic materials is in these foods that your body needs or is harmful to your body.

Even many people and disease physicians are eager to know what elements are in the food they eat every day. This also helps physicians in prescribing different regimens to their patients. For example, for a patient whose body needs iron in large amounts, the physician need to know what foods contain the highest amounts of iron. As we know, good food is the best medicine. Most people who care about their health should refer to a specialist for information about their obesity or overweight or malnutrition and pay a lot of time and money. The current information poverty has caused a lot of people to suffer from diseases and incur a lot of time and costs for curing them.

Most food caused diseases arise due to improper use of feedstuffs around us because people want to meet most of their body's needs by consuming carbohydrates (rice and wheat), despite the fact that most of these materials are converted into fat in body and other needs of the body remain unanswered. This system tells us in which food there are materials required for your body and what foods we should eat to resolve the issue.

\section{BASIC DEFINITIONS}

Health: As defined by World Health Organization, human health includes overall physical health, mental health and community,health, ,(http://en.wikipedia.org/wiki/Health_ system).According to this definition, merely not being sick is not considered as being healthy. According to the World Health Organization definition, the main components of wellness in addition to health and lack of disease include social and economic environment, physical environment, individual's behavior and personality dimensions.

\section{HEALTH CARE}

Health Care includes activities such as diagnoses diseases and their complications, and preventing human diseases and mental disorders [health, 2 January 2015, http://en.wikipedia.org/wiki/Health].

In most cases, medical care is directly related to a country's economy and has allocated a budget of about 9.3 of GDP in OECD member countries. 
Health care is divided to a variety of primary health care or the first category, secondary health care or second category, third category and fourth category.

- Primary health care or first category: related to professionals who advise their patients for the first time and make a connection with them, such as doctors or family physicians.

- Secondary health care or second category: includes service which is provided by those medical specialists who do not have the first contact with the client, such as cardiologist, urologist and dermatologist.

- Third category health care: is related to services that is often assigned to non-patients or people who are referred by primary or secondary medical care and provides facilities for specialized medical examinations and treatment. Some of these types of care include cancer management, neurosurgery, cardiac surgery, plastic surgery, treatment of severe burns, babies advanced services, and other complex medical and surgical interventions.

Fourth category of health care: the fourth category of medical care sometimes is used as a branch of the third category and in fact is considered as a part of the specialized advanced care which requires high levels of specializations which is not widely and easily available, such as some types of unusual methods of diagnosis or surgery. These services are limited in number in any area or in health care centers,(Health_care, 28 February 2015,http://en.wikipedia.org/wiki/Health_care).

\section{HEALTH SYSTEMS}

An organization or person is an institution or entity that will provide health care services to meet the health needs of a community(Health_care / Health care delivery, 28 February 2015).

\section{HEALTH CARE SOFTWARE SYSTEMS}

Health care software systems are mostly focused on physical health and diseases of individuals, than the social, emotional and intellectual aspects of people's health, but with increasing the smart software and information systems, it will also cover this area gradually and it is likely that these systems will cover all the areas of health care.

\section{PREREQUISITES FOR HEALTH CARE SOFTWARE} SYSTEMS

These systems should be web-based and provide online information and services, because health and commu- nity health systems must have powerful notification feature. This will causes the quickly release of data between the people of geographically dispersed regions and for example, if a particular disease spreads in one region or geographical area, without creating panic among the people of the whole country, the people of that area will particularly be notified and medical advice will be reminded to people in order to prevent the rapid spread of the disease; these systems' information must also be constantly updated.

On the other hand, since these systems deal with individual and community health, they should have the maximum accuracy of information, and their decisionmaking systems should have the least mistake; this category of systems are a part of information systems and the heart of every information system is its database, where information are categorized and can be searched and managed. These systems usually need to manage large volumes of information, but the database management systems should be used which are for heavy information management. On the other hand, the type of information used in these software systems are often linked together and sometimes system have to review extensive information for a simple decision.

Database management systems: Database management systems are software systems that are used to manage data and information. In follows, some of the most important of them are listed and analyzed.

SQL-based database management systems:These systems are also known as "Relational" systems and today, are mostly used in information systems. The advantage of these systems is that they communicate appropriately between dispersed and different stored information and in this respect, they are appropriate for the health care systems.

The problem with these systems is that they need a powerful and hardware stable for database management with heavy and high-volume information. Some of the most popular of these systems are Mysql, MS Sql, Oracle $11 \mathrm{~g}$, and Postgresql. The difference between these systems is that some of them, such as Mysql are designed to manage lighter and lower volume information and some of them, such as Oracle are designed to manage information with heavier volume. Also, some database management systems such as Postgresql are appropriate to manage medium-size databases.

SQL language has both structures of Data Definition Language (DDL) and Data Manipulation Language (DML).

NewSQL-based database management systems

NewSql systems are a modern class of relational systems of database management that while maintaining 
the capabilities of relational systems are trying to have a significant speed when dealing with heavy data like Nosql systems and also have the ability of ACID (http:// en.wikipedia.org/wiki/Health_care), (Health_system ,22 January 2015.http://en.wikipedia.org/wiki/Health_system). Some NewSql database management systems include Google Spanner, Clustrix, VoltDB, MemSQL, SAP HANA, Foundation DB, NuoDB, TransLattice, ActorDB [ACID, 20 February 2015,en.wikipedia.org/wiki/ACID).

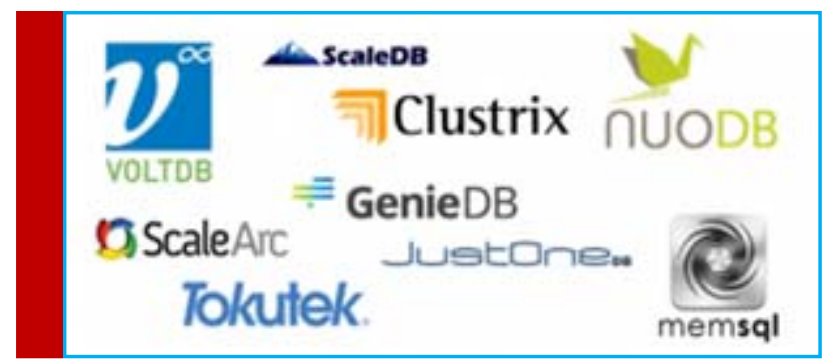

Nosql-based database management systems

Nosql systems that are also interpreted as Not Only SQL systems provide a mechanism to store and retrieve information which is quite different from tabular method of relational systems. The advantage that has made these systems popular is that they are more easily designed and have more horizontal scalability as well as better control and greater access to data [new sql, 18 February 2015 ,http://en.wikipedia.org/wiki/NewSQL]. Data structure used in Nosql systems includes key-value, graph and document which is completely different from the data structure used in Sql systems and this leads to better and faster performance of nosql systems.

Some of the best Nosql systems

There are a variety of systems that use Nosql technology. Some of them are listed below: BaseX, Apache CouchDB, MongoDB, Oracle NoSQL Database, OrientDB, Big Table, Cassandra, Druid, HBase, Hypertable

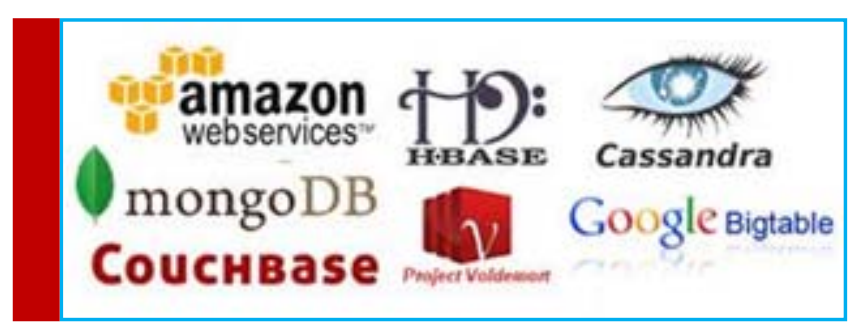

The most appropriate data storage system for comprehensive health system

Given that health care systems or comprehensive health systems should set a lot of facts and items side by side to make decisions and announce the results and examine them, hence nosql systems will not work for these cases and will face difficulty. On the other hand, RDBMS systems, which are also called Traditional DBMS systems are used for programmers and even in universities and are more appreciated. Traditional systems are appropriate and easier for mid- and small-sized works, unless we offset these shortcomings and deficiencies using a more powerful hardware. Hence newsql systems are fit for the design and implementation of such systems and have better responsiveness capability.

\begin{tabular}{|l|c|c|c|}
\hline & Old SQL & NoSQL & NewSQL \\
\hline Relational & Yes & No & Yes \\
\hline SQL & Yes & No & Yes \\
\hline ACID transactions & Yes & No & Yes \\
\hline Horizontal scalability & No & Yes & Yes \\
\hline Performance / big volume & No & Yes & Yes \\
\hline Schema-less & No & Yes & No \\
\hline
\end{tabular}

A comparison between database management technologies (VoltDB (in-memory database) : http://voltdb. $\mathrm{com} /$ )

Capabilities of the comprehensive health system

The capabilities that the comprehensive health system has in order to cover the maximum amount of information are set forth below:

Disease diagnosis

As it was seen in the definition of this system one of the features of this system is disease diagnosis. In medical science, disease diagnosis is based on two types of symptoms:

- Clinical symptoms: are also of two types:

1. Outward symptoms, such as dizziness;

2. Physical tests, such as touching the patient or measuring the fever.

- Paraclinical symptoms: include things such as:

1. Routine tests;

2. Specialized tests;

3. Radiology;

4. Bone marrow samples etc.

Important points in disease diagnosis

- One thing that should be considered when diagnosing a disease is that how often these symptoms of the disease happen.

- Sometimes a symptom indicates different diseases in different genders.

- The amount of symptoms in diseases may include some additional explanations. Example: abdominal pain, where? Does it increase with pressure or decrease? 
- Sometimes during the process of diagnosis, the patient confirms some symptoms which are not related to their original condition, for example, symptoms of osteoporosis and yet declares symptoms related to "cyst" on some part of the body, in other words, a condition sometimes is hidden behind other disease or diseases.

- Sometimes a test should be repeated within a specific time interval.

- Sometimes some symptoms have a higher priority than others.

- In some cases, the symptoms do not indicate a disease; such as hematuria that can occur by eating beets.

- In those who have a history of alcohol or cigarettes, the dose and duration of their use are effective in diagnosing some diseases and prescribing.

- In the diagnosis process, if the system detects the type of disease, it should consider that some diseases shouldn't declare to the patient and should only be referred to a physician, such as cancer and multiple sclerosis.

Amounts of data in testing and during the process of diagnosis:

The system that must receive test data and disease symptoms and examine them should be designed to meet a variety of these experiments data.

Some of the most important of these data are:

- Numerical values: e.g. for glucose levels 200, or blood creatine 1340.

- Values of + and -: e.g. for pregnancy test, blood ph etc.

- Sentential values: e.g. for ultrasound and image.

- Values in the form of file: e.g. a picture or sound for heart examinations."

Offering medical advices

Health and medical care comprehensive system acts like a family physician and is always with people and recognizes the needs of individuals and declares important medical advice to them, when necessary. The system constantly receives people's health information, and people are connected with the system in different ways for hours, and monitor their past and present for the system and this is the system that receives all that are related to the patient's health such as allergies and examines these information constantly and evaluates them over and over again. Not like some doctors who examine all information of their patients for a few minutes and write a prescription for them and prescribe medications sometimes without evaluating them. Examples of these cases are described below:
- The first blood test should be done at age 30;

- If there is a history of diabetes in your family, do the glucose test at the age of 23;

- The breast exams should be done every 6 months after age 30.

Considering and evaluating people's consumption of organic and inorganic matter

One of the unique features of comprehensive health system is that it constantly examines the amount of organic and inorganic matters that people daily consume and if it is harmful for people's fitness as well as their diseases, the system notifies them and determines and recognizes the amount of material required for human body. There are some factors affecting the consumption of organic and inorganic matters, elements and daily calories such as age, weight, sex, addiction, alcohol, smoking, exercise and its type and amount during the day and also fitness. The cases in these topics that have been considered in this system include:

- Controlling food consumption: a lot of minerals and elements are essential for the body and should be consumed on a daily basis in a proportional amount (not less and not more);

- Some materials are not absorbed by the digestive system of some people and have to inject into the body, such as iron or B12 and B6 vitamins.

Comprehensive health system detects these and will not include high levels of these materials in their daily diet.

\section{Medication prescription and its related issues}

Of other features of the comprehensive health system is medicine prescription. The system helps physicians in this way and does a better medicine prescription and away from the errors. Some of the topics that have been studied in this system are as follows:

- Side effects of medicine use;

- Last date of medicine use;

- Side effects of a medicine for an age and weight class;

- Side effects of a medicine for some diseases;

- Allergenic side effects of some medicines;

- Sometimes by seeing a symptom on the patient's body taking the medicine should be discontinued;

- Amount of certain medicines use will vary in cases of using certain foods. For example :

A person with diabetes will take some sugar and sweets; the system determines how much insulin or alternative medicine such as NPH or Nevomix they should consume. 
Study the amount of consuming organic matters and nutrients in people's food

A person, who has a certain diseases, should not daily consume for example, more than 100 grams of a food. This is another issue that comprehensive health system constantly examines. Some topics in this issue include:

- Grade diseases, such as diabetes: for each grade the quantities of medicine vary.

- The amount of different materials consumption in some diseases, if the person is physically active, according to the hours of activity or exercise is different.

Controlling the amount of physical activity

Another feature of comprehensive system health is controlling the amount of physical activity, including sports and jobs of people. Some types of exercise and daily activities or a job are disproportionate for some diseases.

\section{DISEASE TREATMENT}

Different methods to treat a disease

Variety of different methods to treat a disease and the difference in the duration of therapy in any treatment is another issue in examining the comprehensive health system. When prescribing, the comprehensive health system always examines that other than medicine or along with it, are there any other ways to treat a disease with fewer side effects or not? If the disease is treatable with different methods, we reach to this issue that with which of these methods the patient is more comfortable and which will lead to fewer complications and more definitive treatment. Some of these treatments include:

- Medicine

- Sport

- Food

Diseases that people are prone to them

Today, with advances in medical science we can determine people are prone to what diseases and use this information to prevent the development of those diseases. The system detects people who are prone to some diseases and will be able to offer them special cares. Some methods of detecting what people are prone to what diseases include:

- Such as the family history of people in one or more types of diseases.

- Diseases that affected an individual in the past and there is the possibility of recurrence.

- Calculating fitness on the basis of BMI formula.

- Considering some habits in diseases.

- Smoking, alcohol and alcoholic beverages, and drugs.

\section{ENVIRONMENTAL FACTORS}

Environmental factors affect the lives of healthy and sick people. These factors can be useful or harmful for a disease and if this adds to the system, it would help people to choose the best environment to live and work.

\section{CONCLUSION}

There are nearly 90 kinds of major disease that Health care systems prescribe for them. Today, these systems are mostly used as medical assistant but some of these systems, such as comprehensive health system have the ability to learn and become expert and it's not unlikely that one day they decide instead of physicians. Of course, the comprehensive health system will create a whole new world in the field of medical and health and nutrition for people. Now in the third millennium we see physicians making terrible mistakes in diagnosis, prescription and medical advices and yet the field of medicine and medical prescriptions is related to human factors, but what comprehensive health system brings for human fulfills a large part of the definition of World Health Organization and provides great facilities for the first time for public and without any discrimination.

People sit comfortably in their homes and communicate with the system. They declare their medical history, fitness status, feed rate and its type, their job and daily activities, their allergies and whatever is related to their health to the system and from this moment by the minute, the system consider every individual, and declares their needs and helps them to live a healthier life.

Who knows what toxins and how much of them are in a food? Who can tell how for many years people can live? The answer is comprehensive health system.

What can be done to improve people's lives? How much the environmental factors affect people's lives? And many other questions that their answers can be realized only with a scrutiny and long and costly trials which take many years; the comprehensive health system can tell all of this at an insignificant cost to people.

When computers came to assist the accountants for the first time and carried out many complex accounting matters only with a simple click, many people looked at these systems with skepticism and preferred to register and then analyze their daily accounts with accounting books and pens. If today someone is asked to compare between accounting office and computerized accounting systems and assess both of them even if the person lacks the expertise, computer systems will be recommend. Today this system may be criticized, but comprehensive health system will give you so many facilities that you will have no choice but to choose this system. 


\section{REFERENCES}

http://whatis.techtarget.com/definition/entity September 2005,

Health systems, World Health Organization definition, 22 January 2015,http://en.wikipedia.org/wiki/Health_system

Health systems ,Goals, 22 January 2017, http://en.wikipedia. org/wiki/Health_system

Health, 2 January 2015, http://en.wikipedia.org/wiki/Health

Health_care, 28 February 2015,http://en.wikipedia.org/wiki/ Health_care
Health_care,Health care delivery ,28 February 2015, http:// en.wikipedia.org/wiki/Health_care

Health_system ,22 January 2015.http://en.wikipedia.org/wiki/ Health_system ACID, 20 February 2015,en.wikipedia.org/wiki/ ACID

New sql, 18 February 2015, http://en.wikipedia.org/wiki/NewSQL VoltDB (in-memory database) : http://voltdb.com/ 